\title{
Zero modes in the random hopping model
}

\author{
P. W. Brouwer and E. Racine \\ Laboratory of Atomic and Solid State Physics, Cornell University, Ithaca, New York 14853-2501
}

A. Furusaki

Yukawa Institute for Theoretical Physics, Kyoto University, Kyoto 606-8502, Japan

Y. Hatsugai

Department of Applied Physics, University of Tokyo, 7-3-1 Hongo Bunkyo-ku, Tokyo 113-8656, Japan

and PRESTO, Japan Science and Technology Corporation, Kawaguchi-shi, Saitama, 332-0012, Japan

\author{
Y. Morita \\ Department of Applied Physics, University of Tokyo, 7-3-1 Hongo Bunkyo-ku, Tokyo 113-8656, Japan \\ C. Mudry \\ Paul Scherrer Institute, CH-5232 Villigen PSI, Switzerland \\ and Yukawa Institute for Theoretical Physics, Kyoto University, Kyoto 606-8502, Japan
}

(Received 31 January 2002; published 9 July 2002)

\begin{abstract}
If the number of lattice sites is odd, a quantum particle hopping on a bipartite lattice with random hopping between the two sublattices only is guaranteed to have an eigenstate at zero energy. We show that the localization length of this eigenstate depends strongly on the boundaries of the lattice, and can take values anywhere between the mean free path and infinity. The same dependence on boundary conditions is seen in the conductance of such a lattice if it is connected to electron reservoirs via narrow leads. For any nonzero energy, the dependence on boundary conditions is removed for sufficiently large system sizes.
\end{abstract}

DOI: 10.1103/PhysRevB.66.014204

PACS number(s): 71.30.+h, 72.15.Rn, 64.60.Fr, 05.40.-a

\section{INTRODUCTION}

Zero modes, wave functions at zero energy, often arise in problems when a quantum particle moves in a background with a nontrivial topological structure. ${ }^{1-31}$ Quantum fluctuations associated to these zero modes have dramatic physical consequences. They appear both in field theories, related to chiral and parity anomalies, ${ }^{1}$ and in lattice regularization of field theories, ${ }^{2}$ and have applications to a wide range of areas in physics: Chiral symmetry breaking in $(1+1)$-space-time quantum electrodynamics, ${ }^{3}$ edge states along the boundary of a disk threaded by a magnetic flux, ${ }^{4,5}$ singular contributions to the Hall conductance from electrons hopping on a square lattice in the presence of a uniform magnetic field, ${ }^{6}$ superconductivity of a cosmic string, ${ }^{7}$ localization of a fractional charge at a domain wall in a charge-density wave ${ }^{8-13}$ induction of a persistent mass current in ${ }^{3} \mathrm{He}-A,{ }^{14-16}$ antiphase boundaries in narrow-gap semiconductors, ${ }^{17}$ surface $^{18}$ $\left(\right.$ edge $\left.{ }^{19,20}\right)$ states in a superconductor with $d_{x^{2}-y^{2}}\left(+i d_{x y}\right)$ symmetry or in a chiral $p$-wave ${ }^{21}$ superconductor, edge states in nanographite ribbon junctions, ${ }^{22}$ and itinerant-electron ferromagnetism in the repulsive Hubbard model. ${ }^{23}$

Related nontrivial topological structures can also exist in random matrix theory ${ }^{32}$ and in the problem of Anderson localization when the disorder possesses a special symmetry. In this context, an almost half a century old example is that of a one-dimensional chain with link disorder. ${ }^{33}$ Here, zero energy corresponds to the center of the energy band, and an eigenfunction with zero energy is guaranteed to exist if the number of sites in the chain is odd. ${ }^{25}$ In the thermodynamic limit, the density of states ${ }^{33}$ and the localization length ${ }^{34,35}$ are singular at zero energy, whereas correlation functions of the local density of states ${ }^{36,37}$ are algebraic functions. This anomalous behavior is an example of a strongly random critical point. ${ }^{38}$ Its origin is rooted in the stochastic properties of the zero modes supported by the Dirac equation in onespace dimension in the background of a white-noise correlated random mass. ${ }^{36,37,39}$ More recently, a two-dimensional random Dirac Hamiltonian with white-noise correlated $\mathrm{U}(1) \times \mathrm{SU}(N)$ random vector potential was shown to be critical at zero energy. ${ }^{40,41}$ As with the stochastic model of Dyson, this critical behavior can be ascribed to the stochastic properties of zero modes supported by Dirac equations in two-space dimensions in the background of white-noise correlated random vector potentials. ${ }^{42-44}$

While the critical behavior of the (continuum) Dirac equation is related to zero modes in the infinite system, the existence of zero modes for Dyson's stochastic model of a quantum particle hopping on a bipartite lattice with link disorder can also occur for a finite system size. In terms of fermionic creation $\left(c_{i}^{\dagger}\right)$ and annihilation $\left(c_{j}\right)$ operators, the Hamiltonian for this system is

$$
\mathcal{H}=\sum_{i, j} t_{i j} c_{i}^{\dagger} c_{j}
$$

where $i$ and $j$ label the lattice sites on a cartesian grid in $d$ dimensions, say, and the hopping matrix elements $t_{i j}$ are nonzero for nearest neighbors only. Examples of bipartite lattices are depicted in Fig. 1. In general, the $t_{i j}$ will have a 
(a)
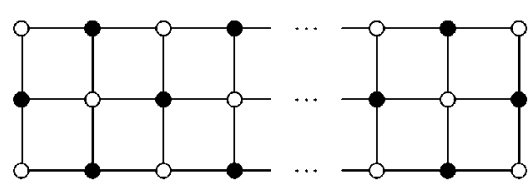

$N=3$

2

1

$\begin{array}{llll}1 & 2 & 3 & 4\end{array}$

$L$

(b)

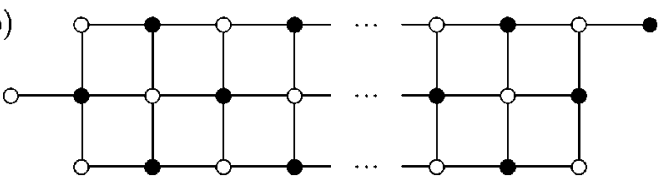

(c)

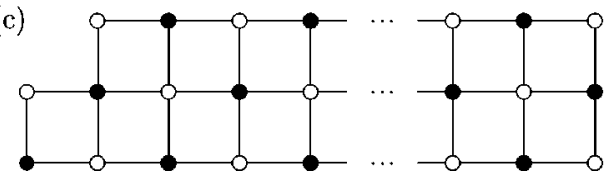

FIG. 1. Three examples of a lattice with different boundary conditions. The sublattices $A$ and $B$ correspond to the white and black sites, respectively. In all three examples, the number $N_{A}$ of sites on sublattice $A$ is one more than the number $N_{B}$ of sites on sublattice $B$. (a) Conventional rectangular shaped wire with $N=3$ and odd $L \gg N$. (b) Boundary conditions with respect to (a) have been changed by adding a white site to the left and adding a black site to the right. (c) Boundary conditions with respect to (a) have been changed by adding a pair of white and black sites to the left.

small random component in addition to an average $t$ which sets the width of the spectrum of $\mathcal{H}$. In this paper we refer to Eq. (1.1) with the random $t_{i j}$ as the "random hopping model." The special case when it is only the phase of the hopping amplitude $t_{i j}$ that is random is also known as the random flux problem.

For the Hamiltonian (1.1), the existence of the zero modes follows from the existence of a "sublattice" (or "chiral") symmetry. This symmetry follows when the lattice is divided into two sublattices $A$ and $B$ such that the hopping matrix $t_{i j}$ only connects sites from the two sublattices, but not sites of the same sublattice. For the example of Fig. 1, the sublattices $A$ and $B$ correspond to the white and black sites, respectively. In a matrix form and after a relabeling of indices, the eigenvalue problem $\mathcal{H}|\psi\rangle=\varepsilon|\psi\rangle$ can be rewritten as

$$
\varepsilon\left(\begin{array}{c}
\psi_{A} \\
\psi_{B}
\end{array}\right)=\left(\begin{array}{cc}
0 & t_{A B} \\
t_{A B}^{\dagger} & 0
\end{array}\right)\left(\begin{array}{l}
\psi_{A} \\
\psi_{B}
\end{array}\right)
$$

where $\psi_{A}$ and $\psi_{B}$ denote the wave function on the lattice sites of the sublattices $A$ and $B$, respectively, and the matrix $t_{A B}$ has the matrix elements $t_{i j}$ with $i \in A$ and $j \in B$. Then, denoting the number of sites in the sublattices $A$ and $B$ by $N_{A}$ and $N_{B}$, counting dimensions in Eq. (1.2) immediately yields that the number of linearly independent zero modes is $\left|N_{A}-N_{B}\right|{ }^{27}$ To see this, note that if $N_{A}>N_{B}, \psi_{A}$ obeys an underdetermined set of linear equations, while $\psi_{B}$ obeys an overdetermined set of equations. (For all lattices shown in Fig. 1, there is one zero mode with support on sublattice $A$.)
Addition or removal of a single site changes the number of zero modes, as it changes the difference $\left|N_{A}-N_{B}\right|$ between the numbers of sites in the two sublattices. At the same time, the singular behavior of the density of states and the localization length in the random hopping model in the thermodynamic limit are considered "intrinsic" properties, i.e., they are derived from continuum models and should not depend on boundary conditions of the lattice. Hence, while both the existence of zero modes for lattices with boundaries and the singular behavior of the localization length are manifestations of the same sublattice symmetry, they are so in very different ways. One might even ask to what extent the localization length of zero modes is representative for the "intrinsic" localization length of the random hopping problem or random flux problem on a lattice without boundaries. This is the question addressed in this paper.

Our answer is that the localization length of the zero modes for lattices with boundaries is not an "intrinsic" property of the random hopping model. After a brief review of the transfer-matrix formalism in Sec. II A, we support this conclusion in Sec. II B by analytical and numerical solution of the problem in the case of a wire geometry: Depending on the boundary conditions, zero modes exhibit a range of localization lengths, the smallest one being of the order of the mean free path. In Sec. II C we then show that this extreme sensitivity to boundary conditions is an anomaly corresponding to the special case $\varepsilon=0$. For any energy $\varepsilon \neq 0$ there is a unique localization length if the system size is sufficiently large. For sufficiently small $\varepsilon$, this unique localization length coincides with the largest of the possible localization lengths at $\varepsilon=0$. We discuss higher dimensional examples in Sec. III and conclude in Sec. IV.

\section{QUASI-ONE-DIMENSIONAL GEOMETRY}

In this section we consider a two-dimensional lattice, $N$ sites wide and $L$ sites long with $L \gg N$. Examples for $N=3$ are given in Fig. 1 whereby sites on sublattices $A$ and $B$ are colored in white and black, respectively. The number of zero modes equals $\left|N_{A}-N_{B}\right|$. For definiteness, we assume $N_{A}$ $>N_{B}$, so that all zero modes have support on sublattice $A$.

In this section, we will compare zero modes in wire geometries with different boundary conditions at the two ends of the wire, as is shown, e.g., in Fig. 1. We will establish that the localization length of these zero modes cannot be thought of as being intrinsic, i.e., independent of the boundary conditions even as the thermodynamic limit $L \rightarrow \infty$ is taken. We will then turn our attention to finite energies and, supported by a numerical solution of the problem, argue that an intrinsic localization length at arbitrarily small but finite energies does indeed exist. The order in which the limits $L \rightarrow \infty$ and $\varepsilon \rightarrow 0$ are taken is thus essential for the extraction of an intrinsic localization length at zero energy.

The sublattice symmetry singles out the band center in that, under an appropriate choice of boundary conditions, an exact energy eigenfunction at that energy can be constructed for any realization of the disorder. This is not true of any finite energy $\varepsilon$ in a closed and finite system. Therefore we will proceed in two steps. We first compare the localization 

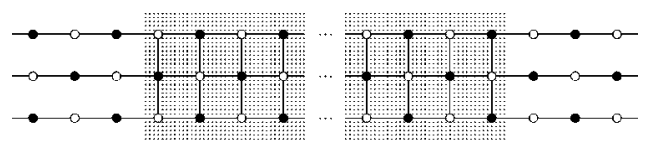

$L$

FIG. 2. Disordered quantum wire of even length $L$ (dotted region) coupled to left and right reservoirs through leads of the same width as the wire. We have chosen leads without transverse hopping for technical convenience.

length of zero modes in a closed system with the exponential decay lengths for transmission probability of plane waves in an open system and establish that they are equal. Then we study how the transmission probability of plane waves is changed when the energy becomes finite. In both steps we use the transfer-matrix formalism, which can deal with open and closed systems in a unified way.

\section{A. Transfer-matrix formalism in an unbounded wire}

\section{Plane-wave representation}

In the absence of disorder, the eigenfunctions of the Schrödinger equation (1.1) in a wire geometry as depicted in Fig. 2 are plane waves. At zero energy, there are $N$ independent wave functions for plane waves traveling to the right, and $N$ independent wave functions traveling to the left. An arbitrary wave function can be expanded in the basis of plane waves. In the presence of disorder, the plane-wave expansion coefficients $a_{n}, n=1, \ldots, 2 N$ acquire a dependence on the position $y$ along the wire. The relation between the $a_{n}(y)$ at different positions along the wire can be expressed through the transfer matrix $\mathcal{M}$,

$$
a_{n}(y)=\sum_{m=1}^{2 N} \mathcal{M}_{n m}\left(y, y^{\prime}\right) a_{m}\left(y^{\prime}\right)
$$

Current conservation and the sublattice symmetry imply that $\mathcal{M}$ can be parametrized as ${ }^{45}$

$$
\mathcal{M}=\left(\begin{array}{cc}
U & 0 \\
0 & U
\end{array}\right)\left(\begin{array}{cc}
\cosh X & \sinh X \\
\sinh X & \cosh X
\end{array}\right)\left(\begin{array}{cc}
V & 0 \\
0 & V
\end{array}\right) .
$$

The $2 \times 2$ grading displayed here is that of right and left moving plane waves. The $N \times N$ matrices $U$ and $V$ are unitary $(\beta=2)$, symplectic $(\beta=4)$, and orthogonal $(\beta=1)$ when the hopping amplitudes are complex, real quaternions, and real valued, respectively. The $N \times N$ matrix $X$ $=\operatorname{diag}\left(x_{1}, \ldots, x_{N}\right)$ is real valued and diagonal. In the absence of the sublattice symmetry, the transfer matrix has a similar parametrization. ${ }^{46}$ The main difference between the cases with and without sublattice symmetry is that without sublattice symmetry the $x_{n}$ can always be chosen positive, while with sublattice symmetry both positive and negative $x_{n}$ appear.

The localization properties of the disordered wire are encoded in the transfer matrix $\mathcal{M}$. They are dominated by the diagonal matrix $X$ in Eq. (2.2). The distribution of the $x_{n}$ at zero energy has been studied in Ref. 45 for the case of a wire with random hopping only. In terms of the mean free path $l$ of the wire, it was found that

$$
x_{n}=\frac{L}{\xi_{n}}, \quad n=1, \ldots, N \text {, }
$$

if $L \gg N l$, up to fluctuations of relative order $(N l / L)^{-1 / 2}$, where the Lyapunov exponents $1 / \xi_{n}$ (the inverse localization lengths) are given by (to leading and subleading order in $N$, see Ref. 47)

$$
\frac{1}{\xi_{n}}=\frac{\beta(N+1-2 n)}{(\beta N+2-\beta) l}, \quad n=1, \ldots, N
$$

For an infinite wire, the conductance is given by

$$
G=\frac{2 e^{2}}{h} \sum_{n=1}^{N} \cosh ^{-2} x_{n} \equiv \frac{2 e^{2}}{h} g
$$

Hence, for an infinite wire it is only the smallest in magnitude of the Lyapunov exponents that governs the exponential decay of the conductance. Its inverse is thus identified with the localization length $\xi$ of the system,

$$
\xi=\left\{\begin{array}{cc}
(\beta N+2-\beta) l / \beta & \text { for } N \text { even } \\
\infty & \text { for } N \text { odd }
\end{array}\right.
$$

As we will find below that all localization lengths $\xi_{n}$ can serve as localization lengths for zero modes in a finite-sized wire, we will refer to their maximum $\xi$ as the localization length for an infinite wire.

\section{Site representation}

An alternative representation for the transfer matrix is obtained using a site representation for the wave function instead of an expansion in plane waves. The sublattice symmetry becomes manifest in the site representation if the wavefunction elements are arranged in $N$-component vectors $\psi_{y}$ containing elements of one sublattice only. In that notation, the Schrödinger equation (1.1) reads

$$
\varepsilon \psi_{y}=T_{y} \psi_{y+1}+T_{y-1}^{\dagger} \psi_{y-1} .
$$

The index $y$ labels the coordinate along the wire and the $N$-component vectors $\psi_{y}$ contain wave-function elements on sites of sublattice $A$ if $y$ is odd and of sublattice $B$ if $y$ is even. A possible choice for the vectors $\psi_{y}$ is shown in Fig. 3. The $N \times N$ matrices $T_{y}$ contain the hopping amplitudes between adjacent sites.

The solution of Eq. (2.7) can be represented in terms of a transfer matrix as well, 


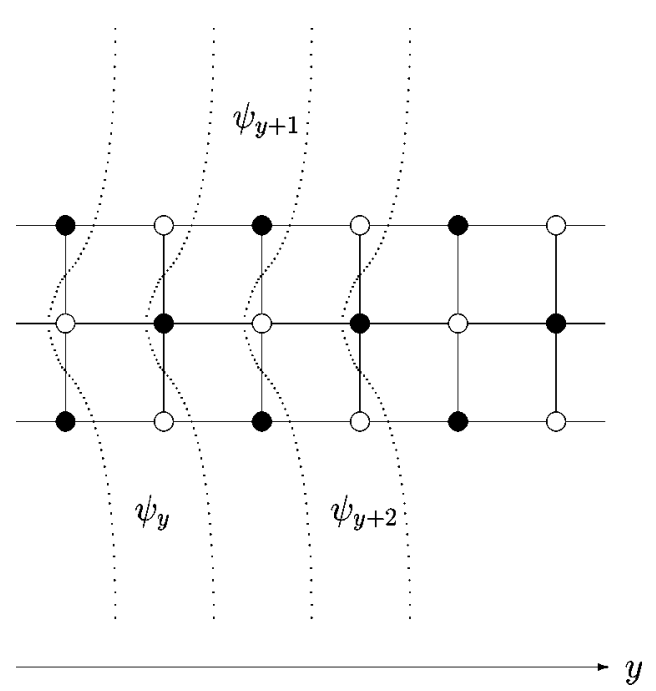

FIG. 3. Choice of the vectors $\psi_{y}$ of Eq. (2.7).

$$
\begin{gathered}
\left(\begin{array}{c}
\psi_{y} \\
\psi_{y-1}
\end{array}\right)=\mathcal{M}\left(y, y^{\prime}\right)\left(\begin{array}{c}
\psi_{y^{\prime}} \\
\psi_{y^{\prime}-1}
\end{array}\right), \\
\mathcal{M}\left(y, y^{\prime}\right)=\prod_{m=y^{\prime}}^{y-1}\left[\left(\begin{array}{cc}
T_{m}^{-1} & 0 \\
0 & 1
\end{array}\right)\left(\begin{array}{cc}
\varepsilon & -T_{m-1}^{\dagger} \\
1 & 0
\end{array}\right)\right] .
\end{gathered}
$$

We note that, if $y-y^{\prime}>0$ is even, the transfer matrix $\mathcal{M}\left(y, y^{\prime}\right)$ is block diagonal at zero energy. In that case, $\mathcal{M}\left(y, y^{\prime}\right)$ can be parametrized as

$$
\mathcal{M}\left(y, y^{\prime}\right)=\left(\begin{array}{cc}
U e^{X} V & 0 \\
0 & T_{y-1}^{-1 \dagger} U e^{-X} V T_{y^{\prime}-1}^{\dagger}
\end{array}\right) .
$$

Here $U$ and $V$ are orthogonal (unitary, symplectic) matrices for $\beta=1(\beta=2,4)$ and $X$ is a diagonal matrix. The grading used in Eq. (2.9) corresponds to the division into sublattices $A$ and $B$. As the transfer matrices of Eqs. (2.8) and (2.1) are related by a simple basis transformation, the distribution of the eigenvalues $x_{n}$ of the matrix $X$ is also given by Eqs. (2.3) and (2.4) above. Hence, as long as we are interested in the Lyapunov exponents only, we can choose freely between the site representation (2.8) and the plane-wave representation (2.2).

\section{B. Bounded wires: Zero energy}

\section{Wave functions}

Zero modes are solutions to Schrödinger equation (1.2) at zero energy. To see how they are constructed, let us first inspect the case of Fig. 1(b) in detail. In this case, the zero mode is a wave function with support on the white sites only. To construct it, we start with three initial values (seeds) for wave function on the three leftmost white sites. We now construct the wave function on all other sites in the same way as one finds the transfer matrix in site representation (2.8): By applying Schrödinger equation to the leftmost black site of the middle row one obtains the value of the wave function on the penultimate leftmost white site of the middle row. Repeating this process column by column for all black sites that have a white site to its right, we can construct a wave function supported on all white sites. For it to be a zero mode, the Schrödinger equation must also be satisfied on the two rightmost black sites that were not used to propagate the wave function on the white sites. For Fig. 1(b) these are the two rightmost black sites. Application of Schrödinger equation on each of those sites yields two linear constraints for the wave function elements on the rightmost white sites. Both constraints can be satisfied since they are implemented linearly on the three seeds of the wave function on the left end.

This example suggests a three-step recursive method to obtain zero modes. First, the zero mode has support on one sublattice only. Second, $N$ independent numbers that make up the $N$-component vector $\psi_{A L}$ are assigned to the values taken by the wave function on the leftmost sites of each row that belong to sublattice $A$. Solution of the Schrödinger equation on all sites of sublattice $B$ except for those without a white nearest neighbor to their left or right (i.e., except for sites of sublattice $B$ that are at the left or right ends of a row) allows to propagate recursively the wave function to the right column by column. The rightmost $N$ values of the wave function thus constructed build the vector $\psi_{A R}$. The relation between the vectors $\psi_{A L}$ and $\psi_{A R}$ that is thus obtained can be expressed as

$$
\psi_{A R}=M \psi_{A L}
$$

The $N \times N$ matrix $M$ is nothing but the counterpart to the upper left block of the transfer matrix in site representation. Hence, by Eq. (2.9), it has the polar decomposition

$$
M=U e^{X} V
$$

where $U$ and $V$ are orthogonal (unitary, symplectic) matrices for $\beta=1 \quad(\beta=2,4)$, and $X$ is a diagonal matrix with eigenvalues $x_{n}$ whose statistics are given by Eqs. (2.3) and (2.4) above.

Third, in order to have a true zero mode, the Schrödinger equation must be obeyed on the remaining $N_{B L}$ and $N_{B R}$ black sites that do not have white nearest neighbors to their left and right, respectively. This gives $N_{B L}$ independent constraints to be satisfied by the elements of $\psi_{A L}$ and $N_{B R}$ independent constraints to be satisfied by the elements of $\psi_{A R}$. As Eq. (2.10) allows for $N$ independent solutions, the number of independent zero modes is equal to

$$
N_{A}-N_{B}=N-\left(N_{B L}+N_{B R}\right) .
$$

The equality $N_{A}-N_{B}=N-\left(N_{B L}+N_{B R}\right)$ followed since the lattice topology is such that the only black sites without left or right white nearest neighbors are located at the far left and far right ends of the wire, respectively. We emphasize that the criterion (2.12) for the existence of zero modes does not rely on the quasi-one-dimensional assumption $N \ll L$ or on the assumption that there be only one "transverse" direction. The only ingredients needed for Eq. (2.12) to hold are the boundary conditions at the end of the disordered region, i.e., the topology of the "wire." The simplifying feature brought by the quasi-one-dimensional limit $N / L \ll 1$, is that the sta- 
tistical properties of the transfer matrix $M$ in Eq. (2.10) are known. We will exploit this knowledge below.

How do the wave-function elements at the left and right ends of the wire compare? To answer this question, we first look at the geometric mean $\phi$ of $\psi_{A L}$ and $\psi_{A R}$,

$$
\phi=e^{+X / 2} V \psi_{A L}=e^{-X / 2} U^{\dagger} \psi_{A R} .
$$

In terms of this geometric mean, the $N_{B L}$ constraints on $\psi_{A L}$ and the $N_{B R}$ constraints on $\psi_{A R}$ can be written in the form

$$
\left(\begin{array}{c}
C_{B L} e^{-X / 2} \\
C_{B R} e^{+X / 2}
\end{array}\right) \phi=0
$$

where $C_{B L}$ and $C_{B R}$ are $\left(N_{B L} \times N\right)$ and $\left(N_{B R} \times N\right)$ matrices with coefficients of order unity, respectively. In the localized regime $L \gg N l$, the $x_{n}$ are spaced by an amount of order $L / N l \gg 1$, so that the coefficients in Eq. (2.14) differ considerably in magnitude. To see what simplifications this brings about, we look at the first row of Eq. (2.14),

$$
\left(C_{B L}\right)_{11} e^{-x_{1} / 2} \phi_{1}+\cdots+\left(C_{B L}\right)_{1 N} e^{-x_{N} / 2} \phi_{N}=0 .
$$

According to Eqs. (2.3) and (2.4), the coefficient $\left(C_{B L}\right)_{1 n} e^{-x_{n} / 2}$ is a random number that fluctuates around $e^{-L /\left(2 \xi_{n}\right)}$. Since by Eq. (2.4) $x_{N}$ is smaller than all other $x_{n}$ by an amount of at least $L / N l \gg 1$, we thus find that the left-hand side of Eq. (2.15) is dominated by the last term, so that we conclude $\phi_{N}=0$, with exponential accuracy. Extending this argument to the first $N_{B L}$ and the last $N_{B R}$ rows of Eq. (2.14) we infer that

$$
\begin{gathered}
\phi_{n}=0, \quad n=N-N_{B L}+1, \ldots, N, \\
\phi_{n}=0, \quad n=1, \ldots, N_{B R},
\end{gathered}
$$

respectively, again to exponential accuracy. Conversely, the only nonzero elements of $\phi$ are $\phi_{n}$ with $n=N_{B R}$ $+1, \ldots, N-N_{B L}$, to exponential accuracy, so that, by Eq. (2.13), the only localization lengths available to the zero modes are $\xi_{n}$ with $n=N_{B R}+1, \ldots, N-N_{B L} \cdot{ }^{48}$ In our notations, a negative localization length corresponds to a wave function exponentially localized near the left end of the wire (since then $\left|\psi_{A L}\right| \gg\left|\psi_{A R}\right|$ in that case), while a positive localization lengths correspond to a wave function exponentially localized near the right end of the wire. A divergent localization length (which can occur for odd $N$ ) signals a zero mode that is critical $\left(\left|\psi_{A L}\right|\right.$ and $\left|\psi_{A R}\right|$ comparable in magnitude to exponential accuracy).

We have verified this scenario by numerical implementation of above recursive construction of zero modes in geometries depicted in Fig. 1 for various choices of $N$ and of boundary conditions. ${ }^{49}$ The agreement found is excellent.

\section{Transmission probability}

A different method to probe the effect of boundary conditions on the localization length of the random hopping model is via the transmission probability of a lattice coupled to electron reservoirs via ideal leads. Boundary conditions

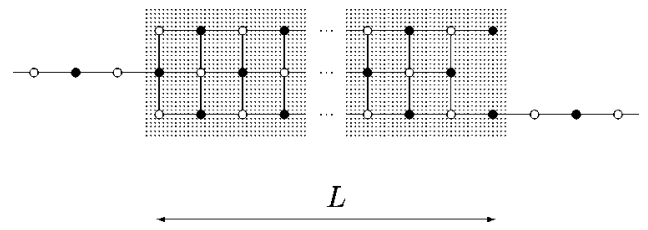

FIG. 4. Example of a disordered quantum wire of even length $L$ (dotted region) coupled to left and right reservoirs via point contacts. In this example the number of left end points on sublattice $A$ is $N_{A L}=2$ and the number of right end points on sublattice $B$ is $N_{B R}=2$. The number of right end points $N_{A R}$ on sublattice $A$ vanishes as does the number of left end points $N_{B L}$ on sublattice $B$. If $L$ is chosen to be odd, $N_{A L}=N_{A R}=2$ whereas $N_{B L}=N_{B R}=0$. When $L$ is odd, a zero mode can only be supported on sublattice $A$ and the conductance must vanish identically.

play a role once the width of the leads is smaller than the width of the sample lattice, as is shown, e.g., in Figs. 4, 5, and 6.

With ideal leads attached to the left and right ends, zero modes with support on sublattice $A$ can coexist with zero modes with support on sublattice $B$. In fact, since a traveling wave at zero energy has support on both sublattices, conductance through the sample is only possible if both types of zero modes exist. ${ }^{50}$ Using the same arguments as for the zero modes in a closed system, the possible localization lengths for zero modes in the presence of leads can be found from counting the number of end points belonging to each sublattice. More precisely, let $N_{B L}$ and $N_{B R}$ be the number of sites of sublattice $B$ without a white nearest neighbor to their left and right, respectively. For all geometries under consideration, $N_{B L}\left(N_{B R}\right)$ match the number of end points on the left (right) end of the wire that belong to sublattice $B$ (sites connected to leads are excluded here). Now, the quasi-onedimensional localization lengths of the zero modes with support on sublattice $A$ are $\xi_{N_{B R}+1}, \ldots, \xi_{N-N_{B L}}$. Similarly, the available quasi-one-dimensional localization lengths for zero modes with support on sublattice $B$ are $\xi_{N_{A R}+1}, \ldots, \xi_{N-N_{A L}}$, where $N_{A L}\left(N_{A R}\right)$ denotes the numbers of end points on the left (right) end of the wire that belong to sublattice $A$ (again, sites connected to leads are here excluded). Whether or not the quasi-one-dimensional limit applies, if $N_{B L}+N_{B R} \geqslant N$ or $N_{A L}+N_{A R} \geqslant N$, there are no zero modes with support on sublattice $A$ or $B$, respectively, and hence no traveling waves and no conductance through the sample. If both $N_{B L}+N_{B R}<N$ and $N_{A L}+N_{A R}<N$, there is a finite conductance through the sample. In that case, the quasi-one-dimensional exponential decay length of the conductance, denoted $\xi^{*}$, is the shorter one of the maximum of the decay lengths for zero-modes on the two sublattices. We give in Tables I and II the values taken by $\xi^{*}$ for the four geometries of Fig. 5 and the three geometries of Fig. 6, respectively.

Thus, we find that the same range of localization lengths shows up in the exponential decay of wave functions in a closed system and of the conductance $G$ in an open system, when the wire is coupled to the electron reservoirs via point contacts. This is in stark contrast to the case of an "infinite" quantum wire (i.e., a wire without point contacts at both ends as depicted in Fig. 2), where only the largest localization 
(a)

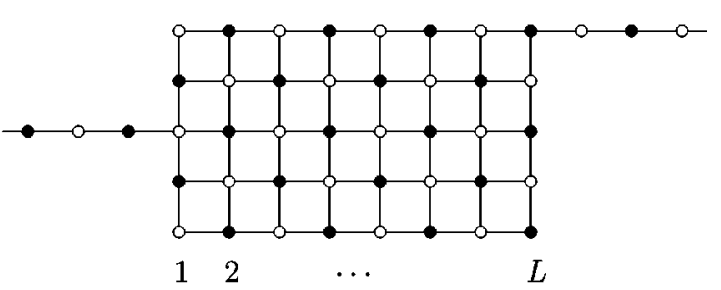

(b)

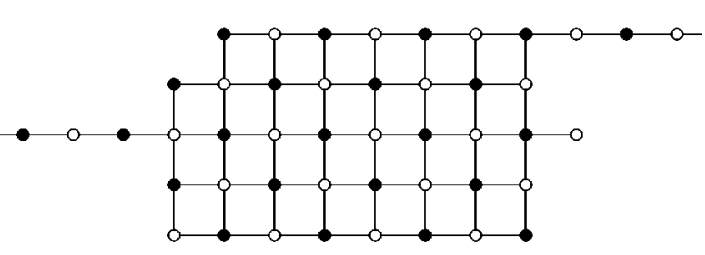

(c)

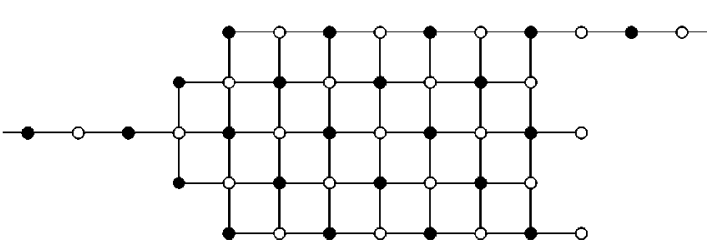

(d)

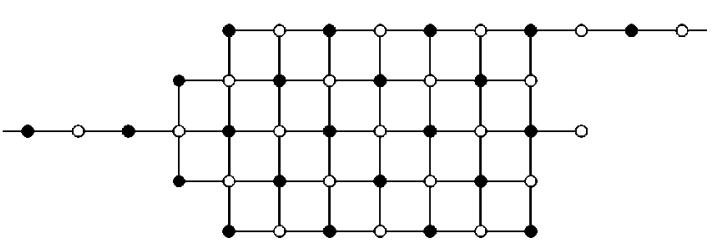

FIG. 5. Four different bounded wires $N=5$ sites wide and $L$ sites long. In all cases $L$ is chosen even and the wire is connected to the reservoirs by single-channel leads. The boundary conditions are specified by (a) $N_{A L}=N_{A R}=N_{B L}=N_{B R}=2$. (b) $N_{A L}=N_{B R}=1$, $N_{B L}=N_{A R}=3$. (c) $N_{A L}=N_{B R}=0, N_{B L}=N_{A R}=4$. (d) $N_{A L}=0$, $N_{A R}=3, N_{B L}=4, N_{B R}=1$.

length determines the conductance. It is also in contrast to the case of a quantum wire with standard diagonal disorder, where the boundary conditions have no effect on the exponential decay length of the conductance.

Again, we have verified this scenario and found excellent agreement between numerics and theoretical expectations.

\section{Bounded wires: Nonzero energy}

To study the importance of boundary conditions at finite energy, we have calculated numerically the conductance of a lattice with random hopping amplitudes and point contacts as a function of energy using the recursive Green-function method. ${ }^{51,52}$ In our numerical simulations we chose realvalued nearest-neighbor hopping amplitudes $t_{i j}$ in the disordered region uniformly and independently in the intervals 1 (a)

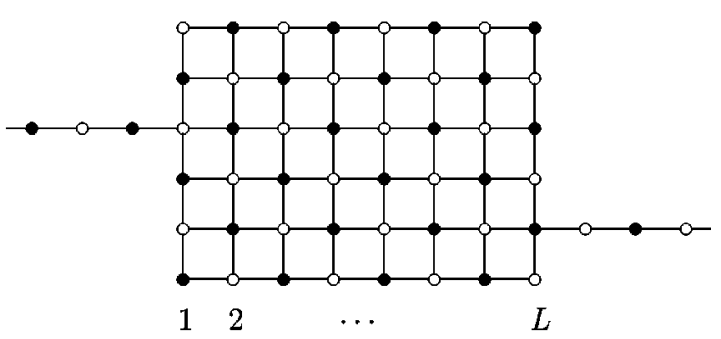

(b)

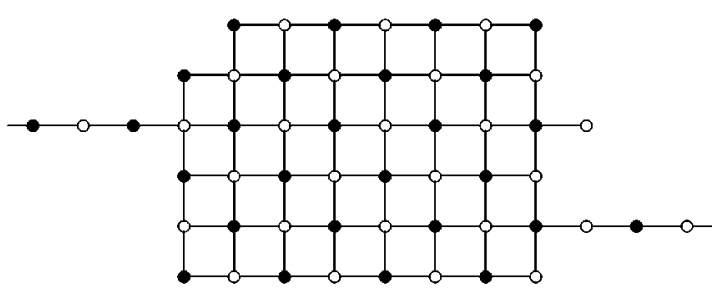

(c)

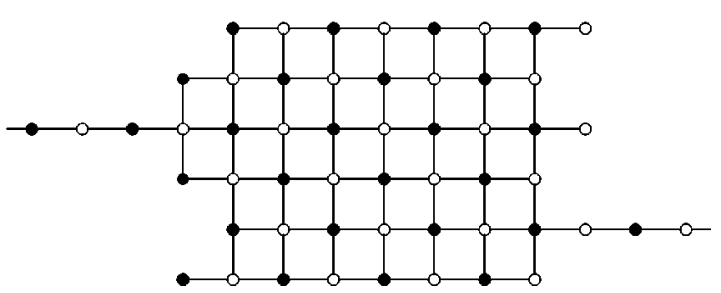

FIG. 6. Three different bounded wires $N=6$ sites wide and $L$ sites long. In all cases $L$ is chosen even and the wire is connected to the reservoirs by single-channel leads. The boundary conditions are specified by (a) $N_{A L}=N_{B R}=2, N_{B L}=N_{A R}=3$. (b) $N_{A L}=N_{B R}=1$, $N_{B L}=N_{A R}=4$. (c) $N_{A L}=N_{B R}=0, N_{B L}=N_{A R}=5$.

$-\delta<t_{i j}<1+\delta$ for hopping in the longitudinal direction and $t(1-\delta)<t_{i j}<t(1+\delta)$ in the transverse direction, with $\delta$ $=0.2$ and $t=0.6$. With this choice the mean free path $l$ is about 65 lattice spacings, as can be estimated from a fit to $\langle\ln g\rangle$ vs $L$ for large $L$ and large energy $\varepsilon\left(\approx 10^{-2}\right)$. In the perfect leads we take $\delta=0$ and $t=0$. The numerical data are obtained after averaging over $10^{5}$ samples. The size of error bars for $\langle\ln g\rangle$ is estimated to be less than $1 \%$. A more detailed account of our implementation of the recursive Greenfunction method can be found in Refs. 53 and 54. The dis-

TABLE I. Maximum localization lengths $\xi_{A}^{*}$ and $\xi_{B}^{*}$, of zero modes on sublattices $A$ and $B$, respectively, for the four different geometries depicted in Fig. 5. The minimum of $\xi_{A}^{*}$ and $\xi_{B}^{*}$, denoted $\xi^{*}$, controls the conductance. The entry $\xi_{A}^{*}=0$ for geometry (d) indicates that no zero mode is supported on sublattice $A$. Correspondingly, the conductance vanishes at zero energy in this geometry as is implied by the vanishing localization length $\xi^{*}=0$.

\begin{tabular}{lccccccc}
\hline \hline Fig. 5 & $N_{A L}$ & $N_{A R}$ & $N_{B L}$ & $N_{B R}$ & $\xi_{A}^{*}$ & $\xi_{B}^{*}$ & $\xi^{*}$ \\
\hline (a) & 2 & 2 & 2 & 2 & $\xi_{3}$ & $\xi_{3}$ & $\infty$ \\
(b) & 1 & 3 & 3 & 1 & $\xi_{2}$ & $\left|\xi_{4}\right|$ & $6 l / 2$ \\
(c) & 0 & 4 & 4 & 0 & $\xi_{1}$ & $\left|\xi_{5}\right|$ & $6 l / 4$ \\
(d) & 0 & 3 & 4 & 1 & 0 & $\left|\xi_{4}\right|$ & 0 \\
\hline \hline
\end{tabular}


TABLE II. Maximum localization lengths $\xi_{A}^{*}$ and $\xi_{B}^{*}$, of zero modes on sublattices $A$ and $B$, respectively, for the three different geometries depicted in Fig. 6. The minimum of $\xi_{A}^{*}$ and $\xi_{B}^{*}$, denoted $\xi^{*}$, controls the conductance.

\begin{tabular}{lccccccc}
\hline \hline Fig. 6 & $N_{A L}$ & $N_{A R}$ & $N_{B L}$ & $N_{B R}$ & $\xi_{A}^{*}$ & $\xi_{B}^{*}$ & $\xi^{*}$ \\
\hline (a) & 2 & 3 & 3 & 2 & $\xi_{3}$ & $\left|\xi_{4}\right|$ & $7 l / 1$ \\
(b) & 1 & 4 & 4 & 1 & $\xi_{2}$ & $\left|\xi_{5}\right|$ & $7 l / 3$ \\
(c) & 0 & 5 & 5 & 0 & $\xi_{1}$ & $\left|\xi_{6}\right|$ & $7 l / 5$ \\
\hline \hline
\end{tabular}

order strength in the numerical calculations presented here is chosen the same as in Ref. 54, so that a comparison of the results is possible.

We have calculated $\langle\ln g\rangle$ vs $L$ for four different boundary conditions for a quantum wire of width $N=5$ and three different boundary conditions for a quantum wire of width $N$ $=6$. The boundary conditions are shown in Figs. 5 and 6 . Our results are shown in Figs. 7, 8, 9, and 10.

Upon increasing the energy away from the band center $\varepsilon=0$, a crossover from the chiral (orthogonal) symmetry class to the standard (orthogonal) symmetry class is expected to take place. For wires without point contacts at the left and right ends, this crossover was studied by three of the authors in Ref. 54. There, we found that the crossover to the standard orthogonal class happens for $\varepsilon \sim \varepsilon_{c}$, where

$$
\varepsilon_{c}=\frac{\hbar v_{F}}{N^{2} l}
$$

is the Thouless energy for a localization volume of linear dimension $\sim N l$. (The relevant localization length is the smaller one of the localization lengths in the chiral and standard symmetry classes). For our calculations we estimate that, with the Fermi velocity given by $v_{F} \approx 2$ in units of the longitudinal mean hopping amplitude, $\varepsilon_{c} \approx 10^{-3}$ for $N=5$ and $N=6$. The largest energy we consider in our calculations, $\varepsilon=10^{-2}$, is well inside the standard orthogonal class, see Fig. 10. For that largest energy, the four or three curves of $\langle\ln g\rangle$ vs $L / l$ in Figs. 7 and 8, respectively, that correspond to the different boundary conditions are indistinguishable. The same conclusion can be reached from Fig. 9, from which one can infer the Lyapunov exponents

$$
\frac{1}{\xi}=-\frac{1}{2} \frac{d\langle\ln g\rangle}{d L}, \quad L \gg N l,
$$

or from Fig. 10, where we showed the energy dependence of $\langle\ln g\rangle$ at a fixed length. This agrees with the conventional understanding that, in the absence of the sublattice symmetry, the localization length is an intrinsic property of the wire, and hence boundary independent.

The two other energies we considered $\left(\varepsilon=10^{-6}\right.$ and $10^{-9}$ ) are both much smaller than $\varepsilon_{c}$, i.e., well inside the chiral class. For short lengths, we see the same strong dependence on boundary conditions that was predicted for the zero modes in the preceding sections. A quantitative verification of the predictions is found from Fig. 9, where the rescaled Lyapunov exponents

$$
-\frac{(N+1) l}{2} \frac{d\langle\ln g\rangle}{d L}
$$

take even integer values for odd $N$ and odd integer values for even $N$ for short $L$ (but still $L \gg N l$ ), in agreement with Eq.
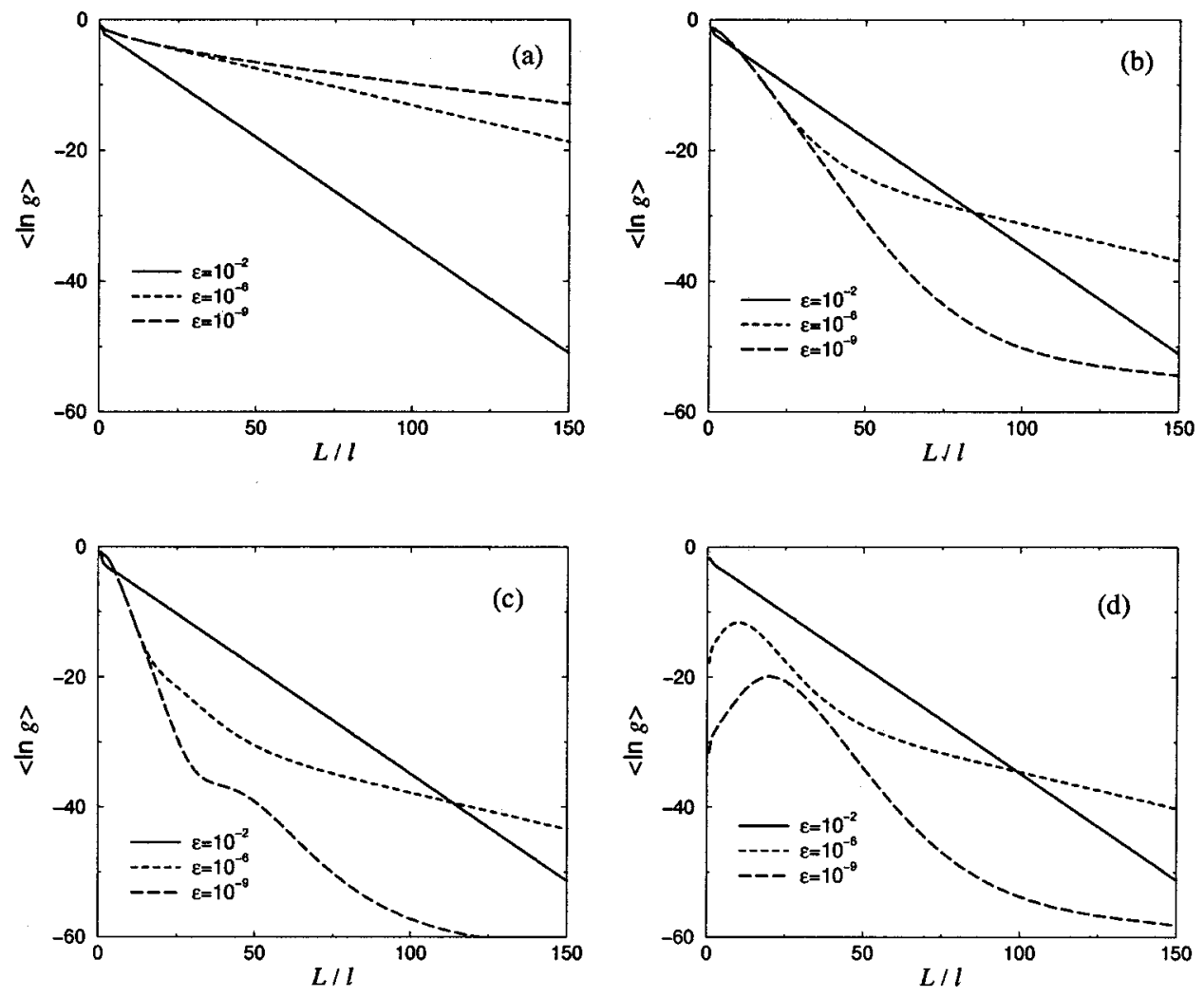

014204-7
FIG. 7. Dependence on the wire length $L, L$ always even, of $\langle\ln g\rangle$ for a quantum wire with random hopping only, connected to the reservoirs via singlechannel ideal leads, and with the boundary conditions specified in Fig. 5. The quantum wire is $N$ $=5$ sites wide. The curves represent different values of the energy $\varepsilon$ and different choices for the boundary conditions at the left and right ends of the wire, as depicted in Fig. 5. 

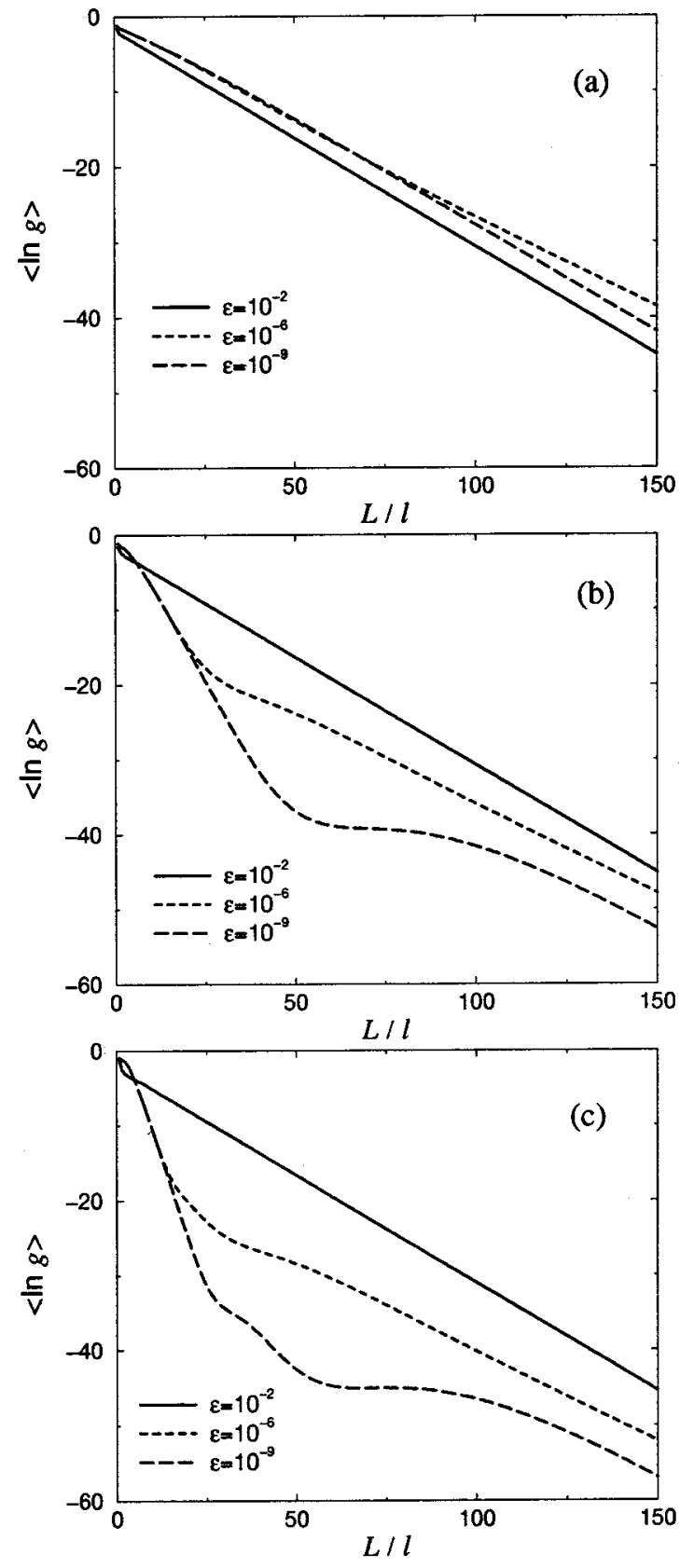

FIG. 8. Dependence on the wire length $L, L$ always even, of $\langle\ln g\rangle$ for a quantum wire with random hopping only, connected to the reservoirs via single-channel ideal leads, and with the boundary conditions specified in Fig. 6. The quantum wire is $N=6$ sites wide. The curves represent different values of the energy $\varepsilon$ and different choices for the boundary conditions at the left and right ends of the wire as depicted in Fig. 6.

(2.4) with $\beta=1$. For larger lengths, however, the dependence on the boundary conditions is lifted, and the Lyapunov exponents are the same for all boundary conditions considered as is illustrated in Fig. 9 at energy $\varepsilon=10^{-9}$. For a sufficiently large length of the wire and upon decreasing the energy, $-(N+1) l d\langle\ln g\rangle / 2 d L$ approaches 0 for $N=5$ and 1 for $N=6$, irrespective of the boundary conditions. Again, this is well illustrated by Fig. 9 at energy $\varepsilon=10^{-9}$. Alternatively, for $N=5(\operatorname{odd} N)$ and energy $\varepsilon=10^{-9},\langle\ln g\rangle$ in Figs.
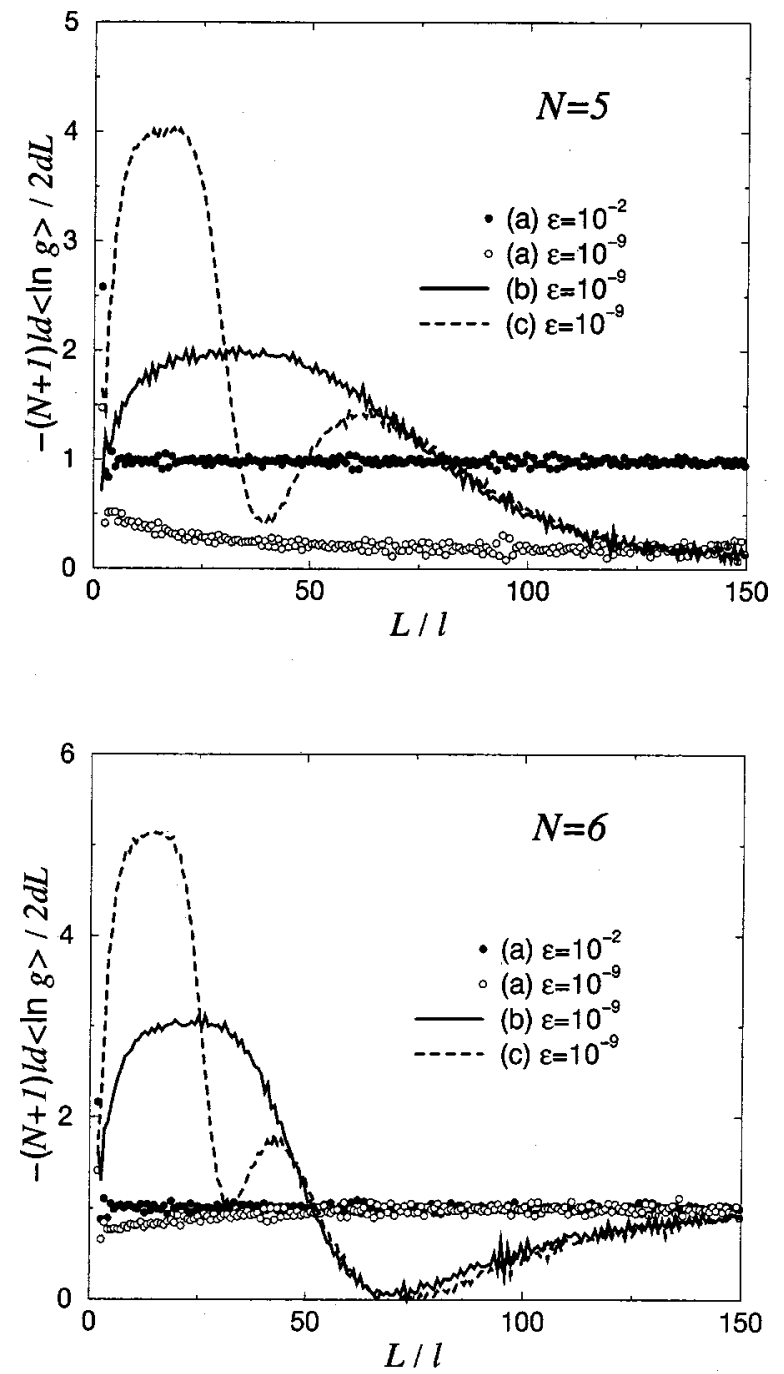

FIG. 9. Lyapunov exponents $d\langle\ln g\rangle / d L$ for the curves shown in Figs. 7 (upper panel) and 8 (lower panel).

7(b) and 7(c) is found to depend linearly on length for small $L$ with a boundary-condition-dependent slope, while $\langle\ln g\rangle$ has a curvature consistent with a $(L / N l)^{1 / 2}$ dependence, as is appropriate for critical conductance statistics. For energy $\varepsilon$ $=10^{-6}$ the $L$ dependence of $\langle\ln g\rangle$ is linear for large $L$, but with a localization length that is significantly larger than for $\varepsilon=10^{-2}$. Such an enhanced localization length is characteristic of the crossover between the chiral and standard classes for a quantum wire without boundaries. ${ }^{54}$ For $N=6$ (even $N$ ) and energies $\varepsilon=10^{-9}, 10^{-6},\langle\ln g\rangle$ in Figs. 8(a)-8(c) decreases linearly with length, but with different slopes for small and large $L$. These slopes correspond to exponential localization controlled by a boundary-condition-dependent zero mode and to exponential localization in the chiral orthogonal class (or, strictly speaking, the crossover between the chiral and standard orthogonal classes) in an infinite wire, respectively. (Note, however, the large energydependent crossover lengths and the nonmonotonous length dependence of the Lyapunov exponents at intermediate length scales in Figs. 7-10.) Hence, from the numerical calculations we conclude that for a finite energy, the Lyapunov 

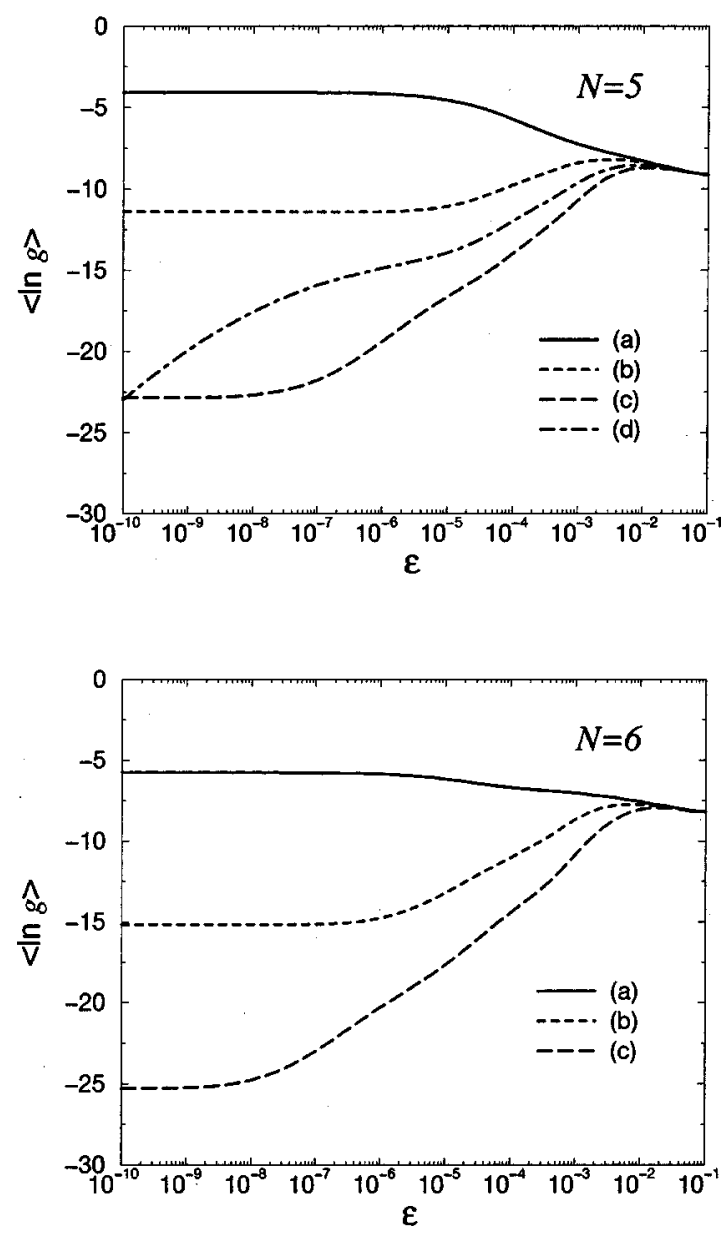

FIG. 10. Crossover as a function of energy for a fixed length $L=20 l$ of the wire of $\langle\ln g\rangle$ for $N=5$ (upper panel) and $N=6$ (lower panel). All traces saturate to different finite values at very low energies except for trace (d) of the upper panel for which the conductance vanishes at the band center.

exponents lose their dependence on the boundary conditions if the wire is sufficiently long. The typical conductance itself, $\exp (\langle\ln g\rangle)$, retains a strong dependence on the boundary conditions for sufficiently long wires through its exponential prefactor as is illustrated by Figs. 7 and 8. However, the slope of $\langle\ln g\rangle$ as a function of $L / N l$ in the regime $L / N l$ $\gg 1$ is independent of the boundary conditions.

The length scales where the Lyapunov exponents begin to cross over from the boundary-condition-dependent value characteristic of the zero modes to the "intrinsic" (smallest) Lyapunov exponent can be estimated as the length scales where the Thouless energy $\varepsilon_{\mathrm{Th}}(L)=g(L) \Delta(L)$ is equal to the energy $\varepsilon$, with $g(L) \sim \exp \left(-2 L /\left|\xi_{n}\right|\right)$ the typical boundary-dependent dimensionless conductance of the wire $(n=1, \ldots, N)$ and $\Delta(L)=\hbar v_{F} / N L$ the mean level spacing of a wire with length $L$. Hence,

$$
L_{\varepsilon, n} \sim\left|\xi_{n}\right| \ln \left(\frac{\hbar v_{F}}{N L_{\varepsilon, n} \varepsilon}\right), \quad n=1, \ldots, N
$$

No useful crossover length can be defined for the boundary condition of Fig. 5(d), where the zero-energy conductance of the wire is zero [see Table I and the downturn of the traces with energies $\varepsilon=10^{-6}, 10^{-9}$ for sufficiently small wire lengths in Fig. 7(d)]. Equation (2.19) implies that the shorter $\left|\xi_{n}\right|$ disappear at shorter wire lengths than the larger $\left|\xi_{n}\right|$. All dependence on boundary conditions is removed and only the smallest of the Lyapunov exponents survives for lengths larger than the second largest of the $L_{\varepsilon, n}$, i.e., beyond $L_{\varepsilon}$ $\equiv L_{\varepsilon,(N-1) / 2}$ for $N$ odd and beyond $L_{\varepsilon} \equiv L_{\varepsilon,(N-2) / 2}$ for $N$ even. This is well illustrated in Figs. 9, where the shortest localization length (corresponding to the highest Lyapunov exponent) disappears first, to be followed by the secondshortest localization length at a wire size that is roughly a factor $2(N=5)$ or 5/3 $(N=6)$ larger (see Tables I and II, respectively).

To summarize, while the exponential decay length of the conductance depends on the boundary conditions for $\varepsilon=0$ even in the limit $L \rightarrow \infty$, the exponential decay of the conductance is governed by the "intrinsic" (largest) localization length $\xi$ for any finite energy $\varepsilon$ different from zero. In this sense, the remarkable dependence of the zero-mode localization lengths and of the zero-energy conductance on boundary conditions can be considered as an anomaly belonging to the case of energy being exactly equal to zero, not as something representative of the thermodynamic limit of the random hopping model.

\section{HIGHER DIMENSIONAL EXAMPLES}

The examples we have discussed so far pertain to a quasione-dimensional geometry. We would like to close with examples of lattices that are extended in two or more dimensions. For the three lattices shown in Fig. 11, three different scenarios apply to the zero-energy conductance between the left and right leads.

For the lattice of Fig. 11(a), the conductance decays exponentially with a decay length of the order of the mean free path of the system as can be seen by direct simulation of the conductance or by constructing recursively zero modes on each sublattices. In the latter case, a seed value is assigned first to the leftmost (rightmost) black (white) site having a pair of white (black) nearest-neighbor sites in the direction orthogonal to the leads. The Schrödinger equation is then solved on the white (black) sites moving to the right (left) column by column. The zero mode on black sites is exponentially localized near the right contact and the zero mode on white sites is exponentially localized near the left contact with a localization length of the order of the mean free path. The exponentially small conductance at the band center is found in all "diamondlike" structures of the form of Fig. 11(a), irrespective of dimensionality and disorder strength.

One possible generalization of the diamondlike geometry of Fig. 11(a) is to couple sites on the lower left and upper right edges of the diamond to the reservoirs. In this case, the number of zero-modes scales with the width of the leads. To each seed on one edge corresponds a zero mode exponentially localized about its $v i s-a ́$-vis on the opposite edge. Linear superposition of these zero modes yield traveling waves on the edges that are exponentially localized in the direction 
(a)

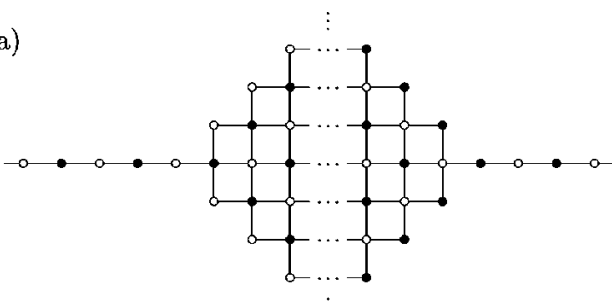

(b)

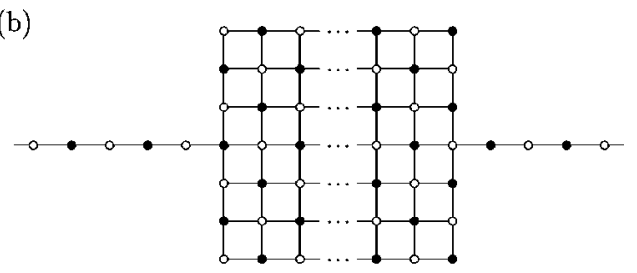

(c)

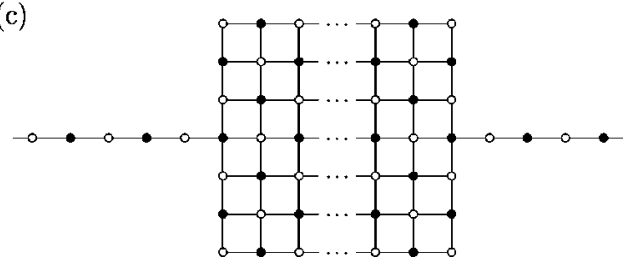

FIG. 11. Three examples of two-dimensional lattices that have different size dependencies of the conductance, see text.

orthogonal to the edges. This is reminiscent of the CallanHarvey effect ${ }^{1}$ in field theory.

For the "square" lattice of Fig. 11(c), the conductance is zero, as this geometry does not allow a zero mode on the black sites according to the arguments of Sec. II B. (Recall that the condition of quasi-one-dimensionality is not needed to establish the conditions for the existence of zero modes.)

Finally, the lattice of Fig. 11(b) has zero modes on white sites and on black sites that are believed to be critical (i.e., not exponentially localized). This is not surprising in a quasione-dimensional geometry for which the lattice only extends in the longitudinal direction, as can be seen using the arguments of Sec. II B. More surprising is that the critical nature of the zero mode seems not to depend on the transversal extension. Vergés in Ref. 55 has studied numerically the conductance distribution in the geometry of Fig. 11(b) for a square lattice made up of up to $799 \times 799$ sites. His conclusion is that the probability distribution in the geometry of
Fig. 11(b) is best fitted by the conductance distribution of a thick quantum wire with an odd number $N$ of channels derived in Ref. 54. He thus concludes that the critical zero mode in the two-dimensional geometry of Fig. 11(b) is quasi-one-dimensional in nature.

\section{CONCLUSIONS}

In this paper we have investigated the dependence on boundary conditions of localization properties of the random hopping problem at the band center and its vicinity. At finite energies, localization properties are intrinsic, i.e., independent of boundary conditions in the thermodynamic limit. Remarkably, this is not true anymore precisely at the band center where both the transmission probability of a disordered region connected to reservoirs by single channel leads and the spatial decay of zero modes in closed systems are highly sensitive to the choice of boundary conditions even as the thermodynamic limit is taken. This sensitivity to boundary conditions was quantified analytically in quasi-onedimensional geometries. In particular, the conditions under which zero modes are critical were given. In higher dimensions, one must rely on numerical simulations to study localization properties of zero modes. However, the task is somehow simplified by an explicit recursive construction of zero modes that we showed applies to a large class of geometries. It is an interesting open problem to determine conditions for criticality of zero-modes beyond quasi-one-dimensional geometries and whether a field-theoretical description of intrinsic critical properties in terms of critical zero modes in the spirit of Refs. 44, 36, 39, and 37 applies to the twodimensional random hopping problem.

\section{ACKNOWLEDGMENTS}

We would like to thank A. Altland for discussions. This work was supported in part by the NSF under Grants No. DMR 0086509 and PHY99-07949 and by the Sloan and Packard foundations (P.W.B.); by Fonds pour la Formation de Chercheur et l'Aide à la Recherche (E.R.); by Grant-inAid for Scientific Research on Priority Areas (A) from the Ministry of Education, Science, Sports and Culture (Grant No. 12046238) (A.F.). P.W.B. and A.F. thank the Institute for Theoretical Physics in Santa Barbara for its hospitality during the final stages of this work. C.M. thanks the Yukawa Institute for Theoretical Physics in Kyoto for its hospitality during the final stages of this work. Numerical data presented here were obtained at the Yukawa Institute Computing facility and the Supercomputing Center, ISSP, University of Tokyo.
${ }^{1}$ C. G. Callan and J. A. Harvey, Nucl. Phys. B 250, 427 (1985).

${ }^{2}$ For a review, see K. Janssen, Phys. Rep. 273, 1 (1996). See also Refs. 6,9,10,17,24-29.

${ }^{3}$ J. Schwinger, Phys. Rev. 128, 2425 (1962).

${ }^{4}$ Y. Aharonov and A. Casher, Phys. Rev. A 19, 2461 (1979).

${ }^{5}$ A. Moroz, Phys. Lett. B 358, 305 (1995).

${ }^{6}$ M. Kohmoto, Phys. Rev. B 39, 11943 (1989).
${ }^{7}$ E. Witten, Nucl. Phys. B 249, 557 (1985).

${ }^{8}$ R. Jackiw and C. Rebbi, Phys. Rev. D 13, 3398 (1976).

${ }^{9}$ W. P. Su, J. R. Schrieffer, and A. J. Heeger, Phys. Rev. Lett. 42, 1698 (1979).

${ }^{10}$ W. P. Su and J. R. Schrieffer, Phys. Rev. Lett. 46, 738 (1981).

${ }^{11}$ H. Takayama, Y. R. Lin-liu, and K. Maki, Phys. Rev. B 21, 2388 (1980); see also R. Jackiw and J. R. Schrieffer, Nucl. Phys. B 
190, 253 (1981).

${ }^{12}$ J. Goldstone and F. Wilczek, Phys. Rev. Lett. 47, 986 (1981).

${ }^{13}$ Z.-B. Su and B. Sakita, Phys. Rev. Lett. 56, 780 (1986).

${ }^{14}$ M. Stone, A. Garg, and P. Muzikar, Phys. Rev. Lett. 55, 2328 (1985).

${ }^{15}$ G. E. Volovik, Pis'ma Zh. Éksp. Teor. Fiz. 43, 428 (1986) [ JETP Lett. 43, 551 (1986)].

${ }^{16}$ M. Stone and F. Gaitan, Ann. Phys. (N.Y.) 178, 89 (1987).

${ }^{17}$ E. Fradkin, E. Dagotto, and D. Boyanovsky, Phys. Rev. Lett. 57, 2967 (1986); Nucl. Phys. B 285, 340 (1987).

${ }^{18}$ C.-R. Hu, Phys. Rev. Lett. 72, 1526 (1994).

${ }^{19}$ G. E. Volovik, Pis'ma Zh. Ékps. Teor. Fiz. 66, 492 (1997) [ JETP Lett. 66, 522 (1997)].

${ }^{20}$ R. B. Laughlin, Phys. Rev. Lett. 80, 5188 (1998).

${ }^{21}$ L. J. Buchholtz and G. Zwicknagl, Phys. Rev. B 23, 5788 (1981).

${ }^{22}$ K. Wakabayashi, M. Fujita, H. Ajiki, and M. Sigrist, Phys. Rev. B 59, 8271 (1999).

${ }^{23}$ E. H. Lieb, Phys. Rev. Lett. 62, 1201 (1989).

${ }^{24}$ D. Weaire and M. F. Thorpe, Phys. Rev. A 4, 2508 (1971).

${ }^{25}$ L. Fleishman and D. C. Licciardello, J. Phys. C 10, L125 (1977).

${ }^{26}$ M. Stone, Ann. Phys. (N.Y.) 155, 56 (1984).

${ }^{27}$ B. Sutherland, Phys. Rev. B 34, 5208 (1986).

${ }^{28}$ M. Inui, S. A. Trugman, and E. Abrahams, Phys. Rev. B 49, 3190 (1994).

${ }^{29}$ Y. Hatsugai, X.-G. Wen, and M. Kohmoto, Phys. Rev. B 56, 1061 (1997); Y. Morita and Y. Hatsugai, Phys. Rev. Lett. 79, 3728 (1997); Phys. Rev. B 58, 6680 (1998).

${ }^{30}$ A. Altland and B. D. Simons, Nucl. Phys. B 562, 445 (1999); J. Phys. A 32, L353 (1999).

${ }^{31}$ O. Motrunich, K. Damle, and D. A. Huse, Phys. Rev. B 65, 064 206 (2002).

${ }^{32}$ D. A. Ivanov, J. Math. Phys. 43, 126 (2002); and references therein.

${ }^{33}$ F. J. Dyson, Phys. Rev. 92, 1331 (1953).

${ }^{34}$ G. Theodorou and M. H. Cohen, Phys. Rev. B 13, 4597 (1976).

${ }^{35}$ T. P. Eggarter and R. Riedinger, Phys. Rev. B 18, 569 (1978).

${ }^{36}$ D. G. Shelton and A. M. Tsvelik, Phys. Rev. B 57, 14242 (1998).
${ }^{37}$ M. B. Hastings and S. L. Sondhi, Phys. Rev. B 64, 094204 (2001).

${ }^{38}$ D. S. Fisher, Phys. Rev. B 50, 3799 (1994); 51, 6411 (1995).

${ }^{39}$ L. Balents and M. P. A. Fisher, Phys. Rev. B 56, 12970 (1997).

${ }^{40}$ A. W. W. Ludwig, M. P. A. Fisher, R. Shankar, and G. Grinstein, Phys. Rev. B 50, 7526 (1994).

${ }^{41}$ A. A. Nersesyan, A. M. Tsvelik, and F. Wenger, Phys. Rev. Lett. 72, 2628 (1994); Nucl. Phys. B 438, 561 (1995).

${ }^{42}$ C. Mudry, C. Chamon, and X.-G. Wen, Nucl. Phys. B 466, 383 (1996).

${ }^{43}$ C. C. Chamon, C. Mudry, and X.-G. Wen, Phys. Rev. Lett. 77, 4194 (1996).

${ }^{44}$ I. I. Kogan, C. Mudry, and A. M. Tsvelik, Phys. Rev. Lett. 77, 707 (1996).

${ }^{45}$ P. W. Brouwer, C. Mudry, B. D. Simons, and A. Altland, Phys. Rev. Lett. 81, 862 (1998).

${ }^{46}$ C. W. J. Beenakker, Rev. Mod. Phys. 69, 731 (1997).

${ }^{47}$ P. W. Brouwer, C. Mudry, and A. Furusaki, Nucl. Phys. B 565, 653 (2000).

${ }^{48}$ Strictly speaking, the above argument does not describe the envelope of the entire wave function, but only the relative magnitudes of the zero mode at the left and right ends of the wire. However, since the very existence depends on the boundary conditions, we can rule out the possibility of a wave function that is localized inside the sample. This conclusion is confirmed by our numerical simulations.

${ }^{49}$ E. Racine (unpublished).

${ }^{50}$ A. Altland and R. Merkt, Nucl. Phys. B 607, 511 (2001).

${ }^{51}$ A. MacKinnon and B. Kramer, Z. Phys. B: Condens. Matter 53, 1 (1983).

${ }^{52}$ H. U. Baranger, D. P. DiVincenzo, R. A. Jalabert, and A. D. Stone, Phys. Rev. B 44, 10637 (1991).

${ }^{53}$ C. Mudry, P. W. Brouwer, and A. Furusaki, Phys. Rev. B 59, 13221 (1999).

${ }^{54}$ C. Mudry, P. W. Brouwer, and A. Furusaki, Phys. Rev. B 62, 8249 (2000); 63, 129901(E) (2001).

55 J. A. Vergés, Phys. Rev. B 65, 054201 (2002). 